\title{
Fractal Landau-Level Spectra in Twisted Bilayer Graphene
}

\author{
Z. F. Wang, ${ }^{\dagger, \dagger}$ Feng Liu, ${ }^{*}$, \\ ${ }^{\dagger}$ School of Physics, Georgia Institute of Technology, Atlanta, Georgia 30332, United States \\ ${ }^{\ddagger}$ Department of Materials Science and Engineering, University of Utah, Salt Lake City, Utah 84112, United States \\ ${ }^{\S}$ Institute of Atomic and Molecular Sciences, Academia Sinica, Taipei 10617, Taiwan
}

\section{Supporting Information}

\begin{abstract}
The Hofstadter butterfly spectrum for Landau levels in a two-dimensional periodic lattice is a rare example exhibiting fractal properties in a truly quantum system. However, the observation of this physical phenomenon in a conventional material will require a magnetic field strength several orders of magnitude larger than what can be produced in a modern laboratory. It turns out that for a specific range of rotational angles twisted bilayer graphene serves as a special system with a fractal energy spectrum under laboratory accessible magnetic field strengths. This unique feature arises from an intriguing electronic structure induced by the interlayer coupling. Using a recursive tight-binding method,

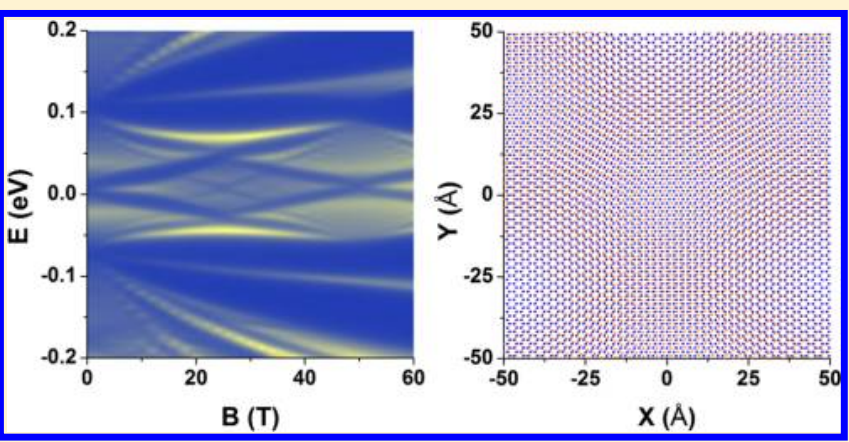
we systematically map out the spectra of these Landau levels as a function of the rotational angle. Our results give a complete description of LLs in twisted bilayer graphene for both commensurate and incommensurate rotational angles and provide quantitative predictions of magnetic field strengths for observing the fractal spectra in these graphene systems.
\end{abstract}

KEYWORDS: Twisted bilayer graphene, interlayer coupling, Landau level, fractal spectra

$\mathrm{T}$ he unique chiral nature of quasiparticles in graphene with a linear dispersion (massless Dirac fermions) results in a novel quantum Hall effect ${ }^{1-3}$ that distinguishes this atomically thin two-dimensional (2D) material from conventional 2D electronic systems. The corresponding Landau levels (LLs) in single-layer graphene are unevenly spaced in energy and proportional to the square root of magnetic field and the LL index $n$. In bilayer graphene, the two atomic layers can be stacked together in many different ways, and a rotation away from the usual Bernal $A B$ stacking by a finite angle will induce a moiré pattern ${ }^{4}$ and modifications of various physical properties. As will be discussed in this Letter, the interlayer coupling will give rise to intriguing changes in the LL spectra that have never been observed in other 2D electronic systems. We will address the evolution of the LLs as a function of the twist angle and the emergence of fractal-like LL spectra in a certain angular range induced by interlayer coupling. Quantitative results will be presented based on tight-binding (TB) calculations that provide an accurate description of the features in the electronic structure of twisted bilayer graphene (TBG) over the entire angular range.

Theoretical interest in $\mathrm{TBG}^{5-14}$ was motivated by the unexpected experimental observation of quasi-ordered nonBernal stacking sequences with different twist angles in the growth of epitaxial graphene on the C-face of SiC. ${ }^{15}$ TBG was also observed in the graphene systems grown by the chemical vapor deposition (CVD) method on transition metals. ${ }^{16-20}$ In these twisted bilayers, the Dirac cones from each layer are rotated by the same angle $\theta$ in momentum space with respect to each other. Interlayer interaction induces coupling between these two sets of linear bands, giving rise to the van Hove singularity near the $M$ point. $^{21}$ First-principles, ${ }^{6,7}$ and tightbinding (TB $)^{5,8}$ calculations have concluded that for a large range of twist angles (larger than $3^{\circ}$ ) TBG preserves linear bands with a renormalized Fermi velocity that decreases with decreasing twist angle as a result of the interlayer coupling. Recent scanning tunneling microscope (STM) measurements have confirmed this velocity renormalization, ${ }^{22}$ although STM measurements on epitaxial graphene layers grown on $\mathrm{SiC}$ only found Fermi velocity values close to that of single-layer graphene. ${ }^{23}$ For twist angles smaller than $3^{\circ}$, the two sets of Dirac cones get close enough to each other that interlayer coupling flattens the linear energy bands and moves the van Hove singularities closer to the Fermi level. This gives rise to a complex band structure around the Fermi level. An increase in localization of the Dirac fermions was suggested both theoretically $^{7}$ and experimentally ${ }^{22}$ for this range of small twist angles.

The large-scale moire pattern introduces new features in the energy spectrum of TBG under magnetic field. It is well-known that the presence of a periodic potential will modify the freeelectron LLs and give rise to fractal-like energy spectra.

Received: May 11, 2012

Revised: June 16, 2012

Published: June 20, 2012 


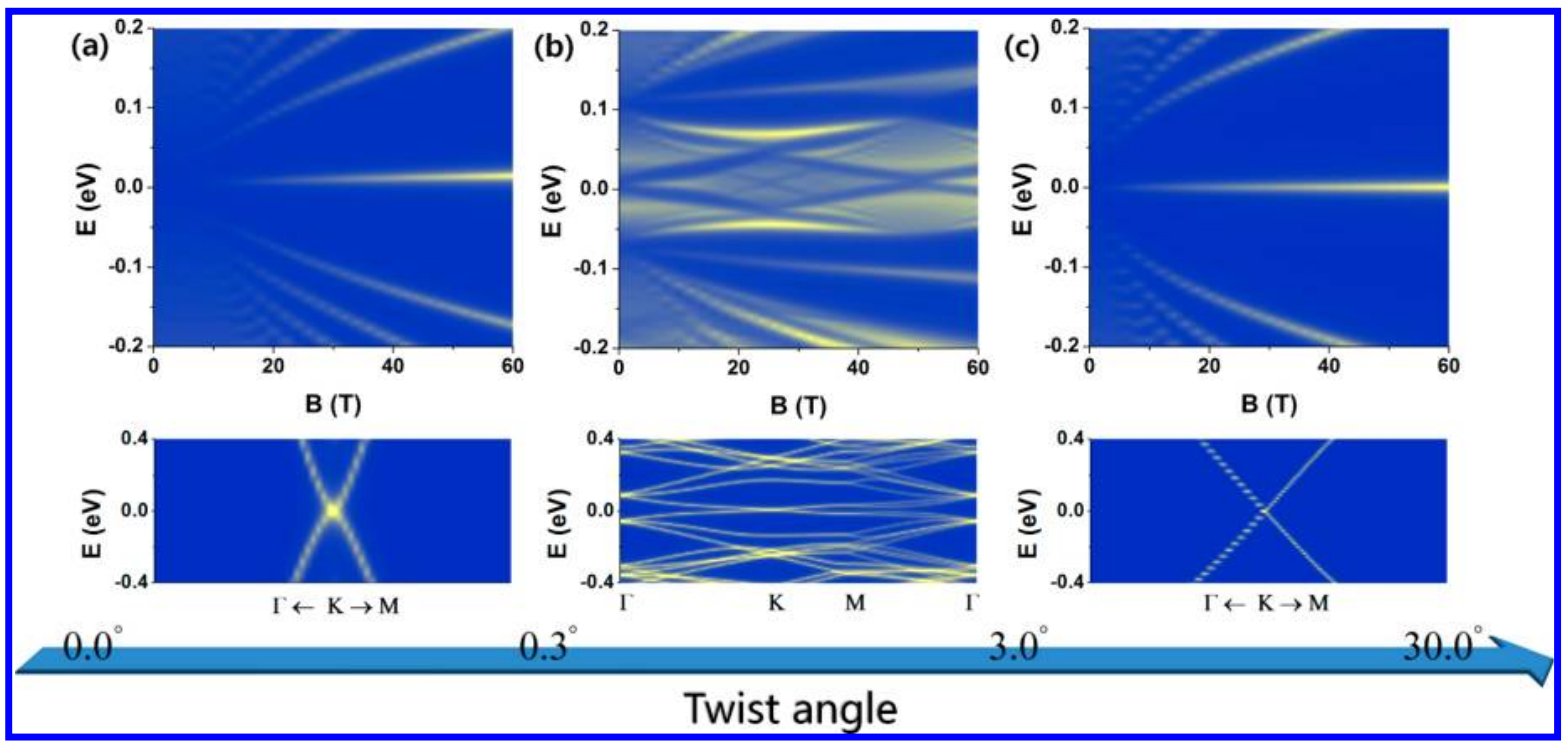

Figure 1. Landau-level spectra for twisted bilayer graphene at three commensurate angles representing three ranges of the twist angle $\theta$. (a) $\theta=$ $0.069^{\circ}$ for the Bernal-bilayer regime $0.0-0.3^{\circ}$, (b) $\theta=1.444^{\circ}$ for the regime $0.3-3.0^{\circ}$, and (c) $\theta=7.565^{\circ}$ for the effectively "single layer" regime $\left(3.0-30.0^{\circ}\right)$. The corresponding electronic bands in these ranges are shown below each spectrum with $\theta=0.0,1.538$, and $7.341^{\circ}$, respectively. The band structures for all the commensurate angles are obtained by diagonalization of the unit-cell Hamiltonian in reciprocal space with periodic boundary condition.

Although the so-called Hofstadter butterfly spectrum has been theoretically proposed in $1976,{ }^{24}$ its experimental realization in a natural system has remained elusive because the required experimental magnetic field is too high $\left(\sim 10^{5} \mathrm{~T}\right)$ for a typical lattice constant of $0.1 \mathrm{~nm}$. One way to circumvent this difficulty was to construct artificial superlattice to increase the lattice constant. $^{25}$ Since there exist superlattices of large lattice constants in TBG that can be tuned by varying the twist angle, this is therefore an opportunity to observe the Hofstadter butterfly in a natural system for the first time.

The analysis of the magneto-electronic spectra has been performed ${ }^{9}$ for TBG using a continuum model to describe the interlayer interaction, and it was concluded that fractal butterfly spectra emerge at laboratory accessible magnetic field strengths. More recently, the energy spectra for a few selected commensurate angles were reported based on a TB calculation with a low-energy approximation, ${ }^{26}$ and similar conclusions were drawn. The interlayer coupling in TBG affects the energy bands in an essential way leading to velocity renormalization and the presence of van Hove singularities, which is more than a simple creation of the moire pattern or a simple introduction of a periodic potential. In particular, for small twist angles the low-energy bands have quite complex characteristics. Our motivation is to accurately map out the complete LL spectra of TBGs as a function of the twist angle, irrespective of their commensuration. Although TBG forms a 2D crystal only at a discrete set of commensurate twist angles, the complete evolution of LLs as a function of the twist angle including incommensurate angles is of fundamental interest. In addition, the Bloch theorem does not apply to incommensurate angles; so direct band-structure calculations are not feasible. For incommensurate twist angles or small commensurate angles where the periodicity becomes quite large, it is a great challenge to carry out the calculations for these LLs. In this Letter, we report computational results of LL spectra in TBG using a realspace recursive TB method with parameters determined by first-principles calculations. We have quantitatively identified three distinct regimes in TBG where the behavior of the LL spectra exhibits characteristics associated with the Bernal bilayer, the Hofstadter butterfly, and a single layer, respectively. Our results give a complete description of LL spectra in TBG and provide a prediction of magnetic field strengths for observing the fractal spectra in the experiment. Comparing to previous studies, we have not only included small angles less than $1^{\circ}$ but also extended the analysis of TBG to incommensurate angles, which gives a better perspective of the LL physics in TBG.

In $\mathrm{TBG}$, the upper layer is rotated relative to the lower one by an angle $\theta$ with $\theta=0^{\circ}$ corresponding to the Bernal-stacked bilayer graphene. The twist angle could be either commensurate or incommensurate. Following the definition by Shallcross et al., ${ }^{6}$ a commensurate angle can be defined by $\theta=\cos ^{-1}\left(\left(3 q^{2}\right.\right.$ $\left.\left.-p^{2}\right) /\left(3 q^{2}+p^{2}\right)\right)$, where $p$ and $q$ are integers. By symmetry, we only need to consider the twist angles within the range of $0-$ $30^{\circ}$. We model TBG in a perpendicular magnetic field by the full TB Hamiltonian ${ }^{27}$

$$
H=\sum_{\mu, v=1,2} \sum_{l, j} t_{\mu l, \nu j} \exp \left[\frac{i e}{\hbar} \int_{\vec{r}_{\mu l}}^{\vec{r}_{\nu j}} \vec{A} \cdot d \vec{l}\right]|\mu l\rangle\langle\nu j|
$$

where $t_{\mu l, \nu j}$ is the hopping parameter, and $\mu$ and $\nu(l$ and $j)$ are the layer (atomic) indices. The magnetic field $B$ is introduced by means of the Peierls substitution $\vec{A}=(0, B x)$. Only $p_{z}$ orbitals are considered, because we are interested in LLs close to the Fermi level. ${ }^{28,29}$ For a finite twist angle, the closest atom in the other layer will not be directly on top of each other. We have used a cutoff distance of $7 \AA$ for the interlayer interaction. The TB parameters are obtained by fitting to energy bands generated from first-principles calculations ${ }^{7}$ (see Figure S1 in Supporting Information) and follow the form of 


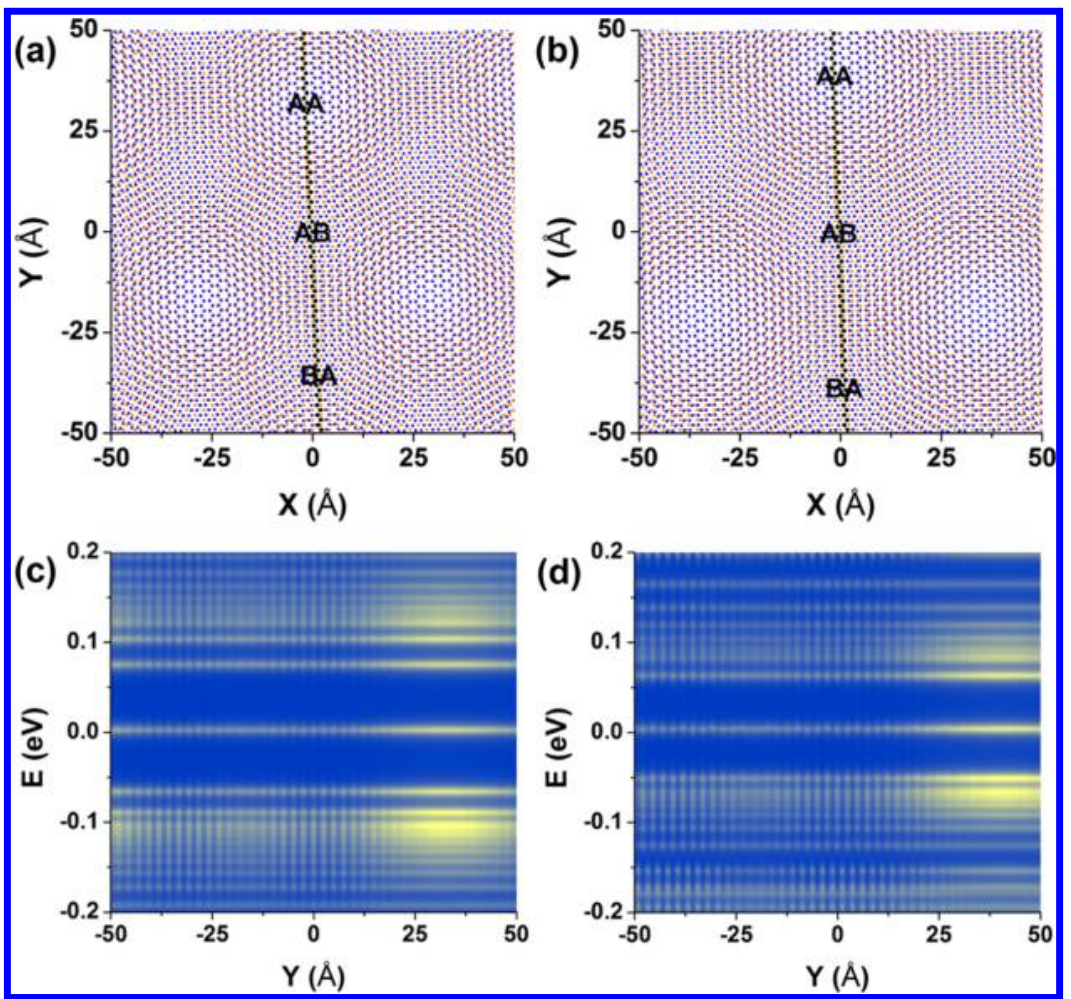

Figure 2. (a) Moiré patterns in twisted bilayer graphene with (a) a commensurate angle $\theta=2.472^{\circ}$ and (b) an incommensurate angle $\theta=2^{\circ}$. AA and $\mathrm{AB} / \mathrm{BA}$ denote different local stacking arrangements. (c,d) The Landau levels (local density of states) at $B=10 \mathrm{~T}$ as a function of location along the lines in (a) and (b), respectively.

$$
t_{\mu l, \nu j}= \begin{cases}\gamma_{1} \exp \left[\lambda_{1}\left(1-\frac{\left|\vec{r}_{\mu l}-\vec{r}_{\nu j}\right|}{a}\right)\right] & (\mu=\nu) \\ \gamma_{2} \exp \left[\lambda_{2}\left(1-\frac{\left|\vec{r}_{\mu l}-\vec{r}_{\nu j}\right|}{c}\right)\right] & (\mu \neq \nu)\end{cases}
$$

van Hove singularities near $\mathrm{M}$ are within $0.1 \mathrm{eV}$ around the Fermi level for $1.538^{\circ}$.) A detailed evolution of the LL spectrum as a function of the twist angle over all three regimes is shown in the movie provided in Supporting Information.

We find that the energy values of the LLs in each regime are almost independent of the location. In addition, similar features are present for close-by commensurate and incommensurate angles. Figure $2 \mathrm{a}, \mathrm{b}$ illustrates the real-space moiré patterns of TBG at two different twist angles, one is commensurate $[\theta=$ $2.472^{\circ}$, Figure $\left.2 \mathrm{a}\right]$ and the other incommensurate $\left[\theta=2^{\circ}\right.$, Figure $2 \mathrm{~b}]$. The former has a large unit cell with a cell area of $\sim 28 \mathrm{~nm}^{2}$. In both cases, regions with atomic stacking patterns of the $\mathrm{AA}$ and $\mathrm{AB} / \mathrm{BA}$ type can be identified. $\mathrm{AA}$ represents the local region where the atoms in the upper layer lie directly above those in the lower layer while $\mathrm{AB} / \mathrm{BA}$ presents the region where the Bernal stacking is found locally. We show the LLs for these two angles with magnetic field $B=10 \mathrm{~T}$ in Figure $2 \mathrm{c}, \mathrm{d}$ along the line as marked in Figure 2a,b, respectively.

The first feature noted in Figure 2 is that for both twist angles where the energy positions of the LLs are independent of the spatial location, as shown in Figure $2 \mathrm{c}$,d. This can be understood in terms of the averaging effect within the magnetic length $\left(l=(\hbar / e B)^{1 / 2} \sim 8 \mathrm{~nm}\right.$ for $\left.10 T\right)$, which is much larger than the graphene lattice constant. Second, the intensity variation of the LDOS is smooth and weakly dependent on the location in the $A A$ region, while in the $A B / B A$ region the LDOS oscillates with the location. This can be understood as a structural effect in that the local atomic environment (coordination) of the $\mathrm{A}$ and $\mathrm{B}$ sublattices is the same in the $\mathrm{AA}$ region but different in the $\mathrm{AB} / \mathrm{BA}$ region. In other words, the local structure of TBG noticeably affects the wave function distribution but not the energy positions of the LLs. In the 

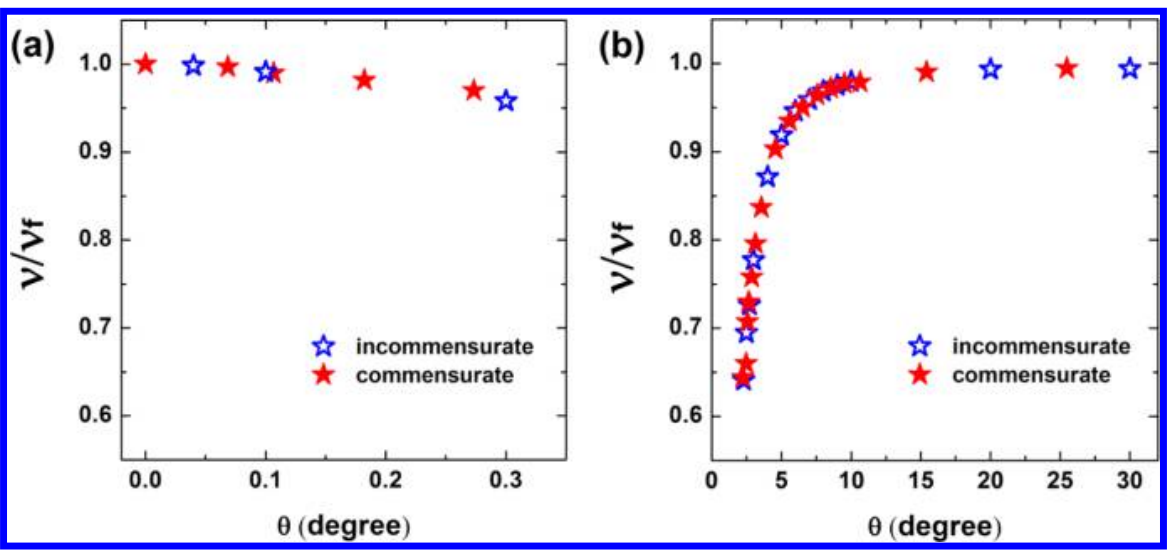

Figure 3. Renormalization of the Fermi velocity as a function of the twist angle in (a) the quasi-Bernal (small $\theta$ ) regime and (b) the effective "monolayer" regime. The velocities have the same meaning in both the quasi-Bernal and effective "monolayer" regimes, but are determined differently by their corresponding LL formulas for Bernal bilayer and monolayer graphene, respectively, as given in ref 31 .
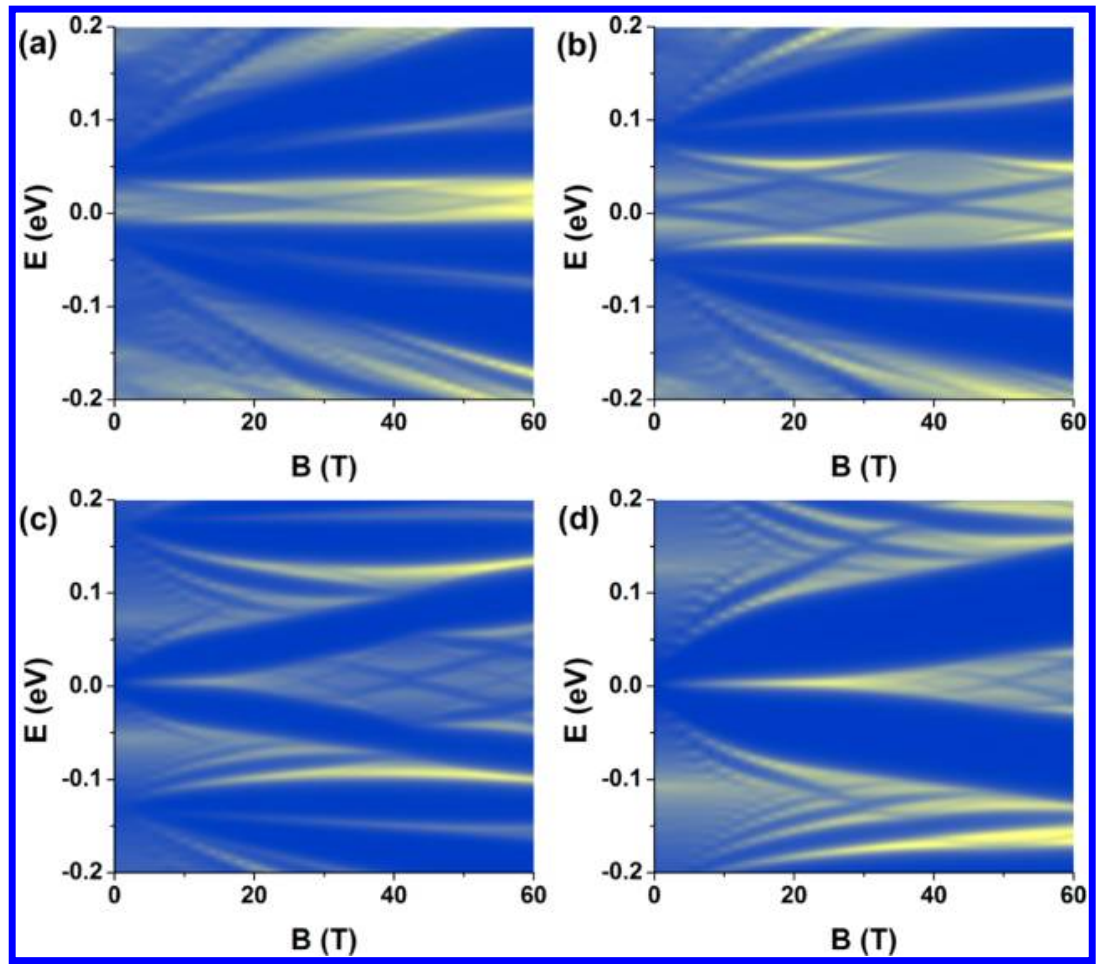

Figure 4. Hofstadter butterfly spectra of twisted bilayer graphene in the intermediate-coupling regime at a few selected twist angles. (a) Commensurate angle $\theta=1.067^{\circ}$, (b) incommensurate angle $\theta=1.3^{\circ}$, (c) commensurate angle $\theta=1.872^{\circ}$, and (d) incommensurate angle $\theta=2.5^{\circ}$.

following, we simply present the LDOS of the LLs as a function of twist angle at one location in TBG.

The electronic structure of TBG with small twist angles (0$0.3^{\circ}$ ) has not been studied previously except for $\theta=0^{\circ}$ (Bernal stacking), because the unit cell is very large in this regime. Our calculated low-energy LLs can be described by the formula for Bernal-stacked bilayer graphene ${ }^{31}$ with a renormalized Fermi velocity $\left(\nu_{\mathrm{F}}\right)$, as shown in Figure $3 \mathrm{a}$. As the twist angle increases, $\nu_{\mathrm{F}}$ slightly decreases. The variation is smooth through both commensurate and incommensurate angles.

For large twist angles $\left(3-30^{\circ}\right)$, the LLs behave like those in single-layer graphene and can be described by a renormalized $\nu_{F}$ using the monolayer formula, ${ }^{31}$ as shown in Figure $3 \mathrm{~b}$. As the twist angle increases, $\nu_{\mathrm{F}}$ rapidly increases initially, and then slowly approaches the value of monolayer graphene at and beyond $\sim 15^{\circ}$. The smooth $\nu_{\mathrm{F}}$ variation shown in Figure $3 \mathrm{~b}$ includes both commensurate and incommensurate angles. The effective interlayer coupling becomes weaker as the twist angle increases, consistent with the perturbation theory of Lopes dos Santos et al. ${ }^{5}$ and experimental observations. ${ }^{22}$

Most interestingly, the LLs in the "intermediate" regime with the twist angles ranging from 0.3 to $3^{\circ}$ display a fractal-like pattern similar to the Hofstadter butterfly, as shown in Figure 4. The Hofstadter butterfly spectra occur in both Bernal bilayer ${ }^{32}$ and monolayer ${ }^{33}$ graphene under very high, experimentally inaccessible field. In contrast, the Hofstadter butterfly spectra in the "intermediate" regime of TBG are apparent at a much smaller field of less than $30 \mathrm{~T}$. As the twist angle increases, the spectrum evolution is continuous going through both commensurate and incommensurate angles as shown in Figure 4 and in the movie provided in Supporting Information. The robustness of the Hofstadter butterfly spectra irrespective of the 
twist angle type should facilitate its experimental observation. Our study shows that the Hofstadter butterfly spectra can also occur in a nonperiodic lattice. In this "intermediate" regime, no simple scaling law is found for the spectra, and the variation is not periodic in $B$ because of the band structure effect. However, the spectral evolution with the twist angle can be qualitatively understood in the following way. As the twist angle increases, the fractal pattern expands in the $E$ axis reflecting an increase of the "renormalized Fermi velocity" (toward the single layer value), while the pattern moves toward larger $B$ fields due to the decrease in unit cell size, so that a larger field is required to yield the same magnetic flux $(\varphi)$. The spatial location independence of the LL spectrum also suggests that the Hofstadter butterfly spectrum can be detected by a local probe such as scanning tunneling spectroscopy as an alternative to transport experiment.

Being a coupled two-layer system, TBG differs from conventional nanofabricated superlattices. If the unit cell size were the only factor that determines the magnitude of $B$ for the occurrence of the Hofstadter butterfly spectra, namely, the larger the superlattice is, the smaller the required $B$ will be, the Hofstadter butterfly would have occurred at even smaller $B$ for angles below $0.3^{\circ}$ where the unit cell size is huge. On the contrary, our results show that the Hofstadter butterfly spectrum only occurs in the "intermediate" regime. This indicates that the interlayer coupling plays a crucial role in the formation of Hofstadter butterfly spectra in TBG. Laissardière ${ }^{7}$ and Morell ${ }^{8}$ showed that for small twist angles $\left(<3^{\circ}\right)$, the bands of TBG near the Fermi level become flat (see Figure 1b) and the electronic states become localized. This special feature resulting from the interlayer coupling seems to also affect the properties of the LLs in the presence of magnetic field.

In summary, we have carried out tight-binding calculations to map out the Landau level spectra in twisted bilayer graphene as a function of the rotational angle and identified three regimes of different features. At large twist angles $\left(>3^{\circ}\right)$, the coupling is the weakest, and the Landau levels are similar to that of singlelayer graphene. At small twist angles $\left(<0.3^{\circ}\right)$, the coupling is the strongest, and the Landau level spectra resemble that of a Bernal-stacked bilayer. At the intermediate twist angles (0.3$3^{\circ}$ ), the electronic energy bands contain flat localized states near Fermi level, and the Landau levels exhibit Hofstadter butterfly fractal features under laboratory-accessible magnetic field strengths. These features are present for both commensurate and incommensurate angles and are believed to arise from the interlayer coupling.

\section{ASSOCIATED CONTENT}

\section{(S Supporting Information}

1. We compare the band structure obtained from the tightbinding calculation with that from first-principles calculations. The parameters of the tight-binding method are determined in order to best mimic the first-principles band dispersions. 2. A brief introduction about the Lanczos recursive method used in our calculation is provided. 3. The calculated results for all angles investigated are shown in a movie. This material is available free of charge via the Internet at http://pubs.acs.org.

\section{AUTHOR INFORMATION}

\section{Corresponding Author}

*E-mail: fliu@eng.utah.edu; meiyin.chou@physics.gatech.edu

\section{Notes}

The authors declare no competing financial interest.

\section{ACKNOWLEDGMENTS}

This work is supported by the Department of Energy (Grants DE-FG02-97ER45632 and DE-FG02-03ER46027). We acknowledge interaction with the Georgia Tech MRSEC funded by the National Science Foundation (Grant DMR-02-05328). We thank the NERSC and CHPC at University of Utah for providing the computing resources.

\section{REFERENCES}

(1) Novoselov, K. S.; Geim, A. K.; Morozov, S. V.; Jiang, D.; Katsnelson, M. I.; Grigorieva, I. V.; Dubonos, S. V.; Firsov, A. A. Nature 2005, 438, 197.

(2) Gusynin, V. P.; Sharapov, S. G. Phys. Rev. Lett. 2005, 95, 146801.

(3) Zhang, Y.; Tan, Y.-W.; Stormer, H. L.; Kim, P. Nature 2005, 438, 201.

(4) (a) Miller, D. L.; Kubista, K. D.; Rutter, G. M.; Ruan, M.; De Heer, W. A.; First, P. N.; Stroscio, J. A. Phys. Rev. B 2010, 81, 125427.

(b) Miller, D. L.; Kubista, K. D.; Rutter, G. M.; Ruan, M.; De Heer, W. A.; Kindermann, M.; First, P. N.; Stroscio, J. A. Nature Phys. 2010, 6, 811 .

(5) Lopes dos Santos, J. M. B.; Peres, N. M. R.; Castro Neto, A. H. Phys. Rev. Lett. 2007, 99, 256802.

(6) Shallcross, S.; Sharma, S.; Pankratov, O. A. Phys. Rev. Lett. 2008, 101, 056803.

(7) De Laissardière, G. T.; Mayou, D.; Magaud, L. Nano Lett. 2010, 10, 804 .

(8) Morell, E. S.; Correa, J. D.; Vargas, P.; Pacheco, M.; Barticevic, Z. Phys. Rev. B 2010, 82, 121407(R).

(9) Bistritzer, R.; MacDonald, A. H. Phys. Rev. B 2011, 84, 035440.

(10) Choi, M.-Y.; Hyun, Y.-H.; Kim, Y. Phys. Rev. B 2011, 84, 195437.

(11) Kindermann, M.; Mele, E. J. Phys. Rev. B 2011, 84, 161406(R).

(12) Xian, L.; Barraza-Lopez, S.; Chou, M. Y. Phys. Rev. B 2011, 84, 075425 .

(13) De Gail, R.; Goerbig, M. O.; Guinea, F.; Montambaux, G.; Castro Neto, A. H. Phys. Rev. B 2011, 84, 045436.

(14) Bistritzer, R.; MacDonald, A. H. Proc. Natl. Acad. Sci. U.S.A. 2011, 108, 12233.

(15) Hass, J.; Varchon, F.; Millán-Otoya, J. E.; Sprinkle, M.; Sharma, N.; De Heer, W. A.; Berger, C.; First, P. N.; Magaud, L.; Conrad, E. H. Phys. Rev. Lett. 2008, 100, 125504.

(16) Kim, K. S.; Zhao, Y.; Jang, H.; Lee, S. Y.; Kim, J. M.; Kim, K. S.; Ahn, J.-H.; Kim, P.; Choi, J.-Y.; Hong, B. H. Nature 2009, 457, 706.

(17) Kwon, S.-Y.; Ciobanu, C. V.; Petrova, V.; Shenoy, V. B.; Bareño, J.; Gambin, V.; Petrov, I.; Kodambaka, S. Nano Lett. 2009, 9, 3985.

(18) Sutter, P. W.; Flege, J.-I.; Sutter, E. A. Nat. Mater. 2008, 7, 406.

(19) Coraux, J.; N'Diaye, A. T.; Busse, C.; Michely, T. Nano Lett. 2008, 8, 565 .

(20) Li, X.; Cai, W.; An, J.; Kim, S.; Nah, J.; Yang, D.; Piner, R.; Velamakanni, A.; Jung, I.; Tutuc, E.; Banerjee, S. K.; Colombo, L.; Ruoff, R. S. Science 2009, 324, 1312.

(21) Li, G.; Luican, A.; Lopes dos Santos, J. M. B.; Castro Neto, A. H.; Reina, A.; Kong, J.; Andrei, E. Y. Nat. Phys. 2010, 6, 109.

(22) Luican, A.; Li, G.; Reina, A.; Kong, J.; Nair, R. R.; Novoselov, K. S.; Geim, A. K.; Andrei, E. Y. Phys. Rev. Lett. 2011, 106, 126802.

(23) Miller, D. L.; Kubista, K. D.; Rutter, G. M.; Ruan, M.; De Heer, W. A.; First, P. N.; Stroscio, J. A. Science 2009, 324, 924.

(24) Hofstadter, D. R. Phys. Rev. B 1976, 14, 2239.

(25) Albrecht, C.; Smet, J. H.; Klitzing, K. V.; Weiss, D.; Umansky, V.; Schweizer, H. Phys. Rev. Lett. 2001, 86, 147.

(26) Moon, P.; Koshino, M. Phys. Rev. B 2012, 85, 195458.

(27) Zhu, W.; Shi, Q. W.; Wang, X. R.; Chen, J.; Yang, J.; Hou, J. G. Phys. Rev. Lett. 2009, 102, 056803.

(28) Wang, Z. F.; Liu, F. ACS Nano 2010, 4, 2459.

(29) Wang, Z. F.; Liu, F. Nanoscale 2011, 3, 4201. 
(30) Wu, S.; Jing, L.; Li, Q.; Shi, Q. W.; Chen, J.; Su, H.; Wang, X.; Yang, J. Phys. Rev. B 2008, 77, 195411.

(31) Jr., J. M. P.; Peeters, F. M.; Vasilopoulos, P. Phys. Rev. B 2007, $76,115419$.

(32) Nemec, N.; Cuniberti, G. Phys. Rev. B 2007, 75, 201404(R).

(33) Dietl, P.; Piéchon, F.; Montambaux, G. Phys. Rev. Lett. 2008, 100, 236405. 